\title{
Computation of Localized Flow for Steady and Unsteady Vector Fields and its Applications
}

\author{
Alexander Wiebel, Christoph Garth and Gerik Scheuermann, Member, IEEE Computer Society
}

(Invited Paper)

\begin{abstract}
We present, extend and apply a method to extract the contribution of a subregion of a data set to the global flow. To isolate this contribution we decompose the flow in the subregion into a potential flow that is induced by the original flow on the boundary and a localized flow. The localized flow is obtained by subtracting the potential flow from the original flow. Since the potential flow is free of both divergence and rotation the localized flow retains the original features and captures the region-specific flow that contains the local contribution of the considered subdomain to the global flow. In the remainder of the paper, we describe an implementation on unstructured grids in both two and three dimensions for steady and unsteady flow fields. We discuss the application of some widely used feature extraction methods on the localized flow and describe applications like reverse-flow detection using the potential flow. Finally, we show that our algorithm is robust and scalable by applying it to various flow data sets and giving performance figures.
\end{abstract}

Index Terms-Flow visualization, Neumann problem, Local contribution, Potential flow.

\section{INTRODUCTION}

$\mathbf{F}$ LOW visualization plays an important role during the design process of all kinds of objects in science and industry. Cars, air planes, turbines, motors and buildings are only few examples. They are very different but for all of them the behavior of flow through or around them can be crucial for durability and usability. Common to all of these objects is that their geometry has large influence on the flow through or around them. Many standard flow visualization techniques ignore this fact completely. They only treat the original velocity field or simple derived fields and thus can miss important features. If, for example, the flow is dominated by a large near-constant component, as is common in the

A. Wiebel and G. Scheuermann are with the Image and Signal Processing Group, University of Leipzig, PF 100920, D-04009 Leipzig, Germany. E-mail: \{wiebel,scheuermann\}@informatik.uni-leipzig.de

C. Garth is with the Computer Graphics \& Visualization Group, University of Kaiserslautern, P.O. Box 3049, D-67653 Kaiserslautern, Germany. E-mail: garth@informatik.uni-kl.de flow past a stationary object, critical points (important for topological analysis) and vortices often do not show up at all although the flow can be more complicated than the velocity field suggests at first glance. A fast, not necessarily near-constant, component of flow through tubes or similar objects can hide the mentioned features in the same way. This is where the geometry comes into play, as the dominant flow most of the time is strongly influenced by the geometry. A bent tube is a simple but intuitive example for this. In cases with such dominant flow the analysis of the flow greatly benefits from removing the hiding component and treating the remaining local component of the flow.

The ideas presented in this paper revolve around the notion of localized flow analysis [1], i.e. the analysis of the contribution in a subregion to the global flow of a given data set. To this purpose, a so-called potential flow is constructed that matches the original field on the boundary of the subdomain but is otherwise simple in the sense that it has vanishing divergence and curl. In other words, it represents the laminar flow in the subdomain, which is induced by the geometry of the domain and the conditions on its boundary [2]. By subtracting this field from the original flow (see Fig. 1), we are left with a localized flow that is confined to the subdomain under consideration and contains the local contribution to the global flow. Visualization methods that are based on divergence or rotation of the flow (both local in nature ${ }^{1}$ ) are unaffected by this approach since the localized flow retains the original rotation and divergence. Methods based on the velocity field are able to detect features in localized flow which were hidden in the original flow.

The method presented here works well for both twodimensional and three-dimensional flow fields and is even extensible to unsteady flows. The choice of subregion is arbitrary up to the condition that it is a simple domain with piecewise smooth boundary. We describe an

\footnotetext{
${ }^{1}$ One reviewer had concerns about the notion of rotation and divergence being local. See Appendix I for a dicussion of his example problem.
} 


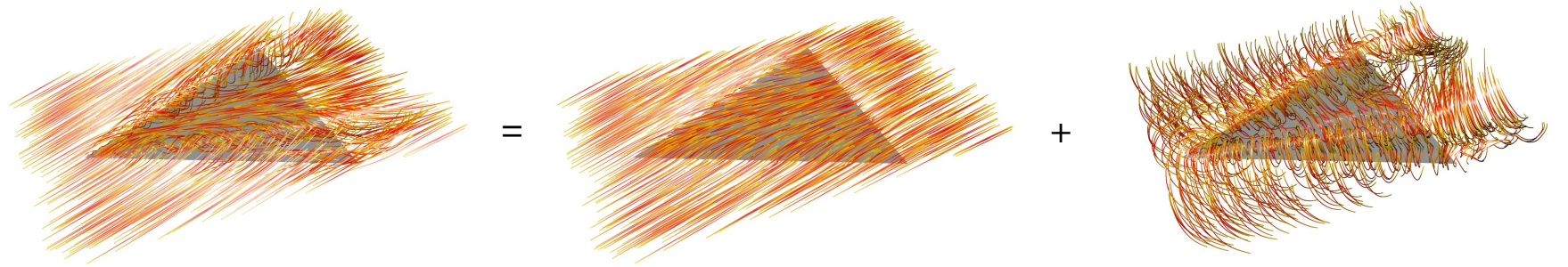

Fig. 1. Illustration of the different components of the flow around the delta wing in EDELTA data set. Left: Original flow field from the CFD simulation, Middle: Potential flow computed by the original field's flow normal to the boundary. Note that the flow is simple but not constant. Right: Localized or region-specific flow obtained by subtracting the potential flow from the original field.

improved version of the algorithm presented in [1] which implements the given ideas on unstructured triangular or tetrahedral meshes using a finite-element approach and extend it for the use with time-dependent fields. Although the computation of the potential flow is a complicated numerical procedure, our algorithm works well even on large CFD data sets with millions of cells.

Our work can be seen as having similarities to what others have published before (cf. [3]-[5]), therefore we describe some essential differences to the work presented here in Section II as well as other work that is related to this paper. In Section III, we recall the mathematical concepts that the region-specific flow is based on and extend these concepts to fit the time-dependent case in Section IV. We give a detailed discussion on the usefulness of the region-specific and potential flow for data set analysis in Section V. The implementation on triangular and tetrahedral grids is the topic of Section VI. Finally, we discuss the application on some examples in Section VII. Section VIII concludes on the presented work.

\section{RELATED WORK}

The notion of localized flow analysis under preservation of the original characteristics of the field (i.e. divergence and rotation) is in part related to work published by Polthier and Preuss [3], [4] (in 2D) and Tong et al. [5] (in 3D). These authors employ the Hodge decomposition theorem from vector analysis, stating that any vector field can be decomposed into three fields containing the divergence, rotation and harmonic parts. The decomposition is given in terms of potentials for the divergence- and rotation-components,

$$
\mathbf{v}=\operatorname{grad} u+\operatorname{curl} \mathbf{w}+\mathbf{h},
$$

which are computed explicitly. Analysis is then attempted by locating features as extremal points of the first two components. Although these approaches seem quite similar to what we describe here, our motives and technique are different. It is our aim to analyze the localized flow with conventional flow visualization techniques, as opposed to making use of the potentials for that purpose. Moreover, in spite of the superficial similarity between the potential flow and the harmonic field $\mathbf{h}$ from above, we believe that our approach is better suited to the localized analysis of flow since we use specific boundary conditions to guarantee that the potential flow contains the part of the flow that does not originate in the considered domain. No such condition is imposed on $\mathbf{h}$. Last but not least, the computation of the potential flow is conceptually simpler than that of $u$ and $\mathbf{w}$, as only one potential and this only of scalar nature has to be computed.

Concerning topological analysis and feature extraction of vector fields, there is a large body of literature available. Post et al. [6] provides a good overview. Of special interest in this paper are topological methods as treated by many authors, e.g. Helman and Hesselink [7], Globus [8], Scheuermann et al. [9], Tricoche et al. [10] and Theisel et al. [11] to name just a few. We are also concerned with more general feature extraction methods, such as the vortex core line extraction method of Sujudi and Haimes [12] and the region-based $\lambda_{2}$-criterion by Jeong and Hussain [13] that we discuss in the context of the localized flow. As a fast moving frame of reference is a simple example for a dominating flow component hiding vortices, the work of Sahner et al. [14] concerning a vortex core extraction method that is independent of the frame of reference is of interest. The method computes ridge and valley lines of Galilean invariant vortex region quantities, such as the mentioned $\lambda_{2}$-criterion to extract the vortex core lines. Stegmaier et al. [15] combine the $\lambda_{2}$-criterion with the method of Banks and Singer [16] to extract Galilean invariant but distinct regions for individual vortices.

There is some recent work by Laramee et al. [17] 
which presents a simple method for the extraction of regions of reverse flow (or recirculation). They investigate data where the main flow should go from negative to positive $x$-coordinate and regard regions containing vectors with negative $x$-component as reverse flow zones. Obviously, this idea can be applied to arbitrary main flow directions (different from $x, y$ and $z$ ) by evaluating the dot product of the vectors with the respective direction and testing for negativity. However, for this method the main flow direction has to be known a priori and it has to be constant all over the dataset. As we will see later, these problems can be circumvented using the potential flow computed by our method.

\section{LOCALIZED FLOW COMPUTATION}

In the following, let $\mathbf{v}: \mathbb{R}^{d} \rightarrow \mathbb{R}^{d}, d=2,3$ be a continuous (flow) vector field. Let $\Omega \subseteq \mathbb{R}^{d}$ be an open, bounded and connected domain and $\mathbf{n}$ the outward normal field on $\partial \Omega$.

In order to analyze the specific contribution of the flow in $\Omega$ to the global flow field, we define the regionspecific flow $\mathbf{v}_{R}: \Omega \rightarrow \mathbb{R}^{d}$ by requiring two essential conditions:

1) it retains the essential behavior of the flow in terms of rotation and divergence, i.e.

$\operatorname{div} \mathbf{v}_{R}=\operatorname{div} \mathbf{v} \quad$ and $\quad \operatorname{curl} \mathbf{v}_{R}=\operatorname{curl} \mathbf{v} \quad$ on $\Omega$.

2) it is isolated from the global flow on the boundary of the subdomain, i.e. the region-specific flow through the boundary vanishes:

$$
\mathbf{v}_{R} \cdot \mathbf{n}=0 \text { on } \partial \Omega .
$$

The suitability of these conditions is discussed in more detail in Section V. The difference of global and regionspecific flow is then given by

$$
\mathbf{v}_{P}:=\mathbf{v}-\mathbf{v}_{R}
$$

Owing to the linearity of divergence and curl, $\mathbf{v}_{P}$ must satisfy

$$
\operatorname{div} \mathbf{v}_{P}=0 \text { and } \operatorname{curl} \mathbf{v}_{P}=0 \text { on } \Omega
$$

and we find that

$$
\mathbf{v}_{P} \cdot \mathbf{n}=\mathbf{v} \cdot \mathbf{n} \quad \text { on } \partial \Omega \text {. }
$$

We next look at how the construction of $\mathbf{v}_{P}$ can be achieved by a simple mathematical procedure.

\section{A. A Special Neumann Problem}

Let us assume that $\mathbf{v}_{P}$ is given as the gradient of a function $u: \Omega \rightarrow \mathbb{R}$ (then $\mathbf{v}_{P}$ is called potential flow). It is immediate that

$$
\operatorname{curl} \mathbf{v}_{P}=\operatorname{curl} \operatorname{grad} u=0 \quad \text { on } \Omega .
$$

Requiring that $\mathbf{v}_{P}$ has vanishing divergence, we compute

$$
0=\operatorname{div} \mathbf{v}_{P}=\operatorname{div} \operatorname{grad} u=\Delta u \quad \text { on } \Omega,
$$

where $\Delta$ denotes the Laplace operator on scalar functions. Rewriting Eq. (2) in terms of $u$, it turns into

$$
\mathbf{n} \cdot \operatorname{grad} u=\mathbf{v} \cdot \mathbf{n} \quad \text { on } \partial \Omega .
$$

Hence, for $\mathbf{v}_{P}:=\operatorname{grad} u$ to fulfill the conditions (1) and (2), $u$ must solve

$$
\begin{aligned}
\Delta u & =0 & & \text { on } \Omega \\
\mathbf{n} \cdot \operatorname{grad} u & =\mathbf{v} \cdot \mathbf{n} & & \text { on } \partial \Omega
\end{aligned}
$$

This class of problem is called a Neumann-Laplace problem for $u$, and it is uniquely solvable up to a constant. From this construction, we are able to determine $\mathbf{v}_{P}$ by solving for $u$. Since we are only interested in grad $u$, the constant is essentially factored out and does not influence the result. The region-specific flow is then given by

$$
\mathbf{v}_{R}:=\mathbf{v}-\operatorname{grad} u .
$$

A unique solution to (3) and (4) can be obtained by requiring

$$
\int_{\partial \Omega} \mathbf{v} \cdot \mathbf{n}=0
$$

implying that the total flow through the boundary must vanish. This ensures that the right hand side is in the orthogonal complement of the kernel of the Laplacian with pure Neumann boundary conditions. The compatibility condition (5) is a-priori fulfilled for incompressible flows (e.g. liquid flow), since by Stokes' theorem

$$
\int_{\partial \Omega} \mathbf{v} \cdot \mathbf{n}=\int_{\Omega} \operatorname{div} \mathbf{v}=0 .
$$

In the next section, we detail a modification of the Neumann problem for the case of compressible flows.

\section{B. Compressible Flows}

When considering compressible flows, e.g. those arising as solutions of the full Navier-Stokes equations, the compatibility condition (5) does not necessarily hold. 
However, we note that compressible flows satisfy the continuity equation ${ }^{2}$.

$$
\frac{\partial \rho}{\partial t}+\operatorname{div} \rho \mathbf{v}=0
$$

where $\rho>0$ denotes a material density that may vary spatially. Based on this, we are able to enhance our approach from above to guarantee results for steady compressible flows. We propose a modified Neumann problem in the form

$$
\begin{aligned}
\Delta u & =0 & & \text { on } \Omega \\
\mathbf{n} \cdot \operatorname{grad} u & =(\rho \mathbf{v}) \cdot \mathbf{n} & & \text { on } \partial \Omega .
\end{aligned}
$$

As for steady flows $\frac{\partial \rho}{\partial t}=0$, the compatibility condition for this system coincides with Eq. (6) and is hence fulfilled. Then, $\mathbf{v}_{P}$ is again divergence- and curl-free and by setting

$$
\rho \mathbf{v}_{R}:=\rho \mathbf{v}-\operatorname{grad} u
$$

it follows

$$
\mathbf{n} \cdot \rho \mathbf{v}_{R}=\mathbf{n} \cdot(\rho \mathbf{v}-\operatorname{grad} u)=0 \quad \text { on } \partial \Omega .
$$

Dividing by $\rho$ we find

$$
\mathbf{n} \cdot \mathbf{v}_{R}=0 \text { on } \partial \Omega,
$$

i.e. the region-specific flow is again confined to $\Omega$ and inherits the characteristics of the original flow.

\section{Extension to Time-DEPEndent CASE}

As the notion of localized flow can be formulated with two purely mathematical conditions (see Sec. III), it is easily extended to unsteady flow.

In the steady case the first condition requires that the localized flow exhibits the same divergence and vorticity as the original flow. Lifting this condition to the unsteady case it says that the localized flow has to have the mentioned divergence and vorticity all the time. As, now, the computation of divergence and vorticity do not depend on time in any way, this just means that the condition from the steady case has to be fulfilled for every instantaneous time step of the unsteady field.

Proceeding from the theoretical considerations to practical CFD datasets, we find that only few instantaneous time steps are given. The fields between these steps have to be interpolated. Fortunately, the following considerations show that if we have two time steps fulfilling the first condition for the localized flow all fields obtained by linear interpolation fulfill the same condition.

Let $\mathbf{v}_{R}^{i}$ and $\mathbf{v}_{R}^{j}$ be two fields that, together with two time steps $\mathbf{v}^{i}$ and $\mathbf{v}^{j}$ of the original field, satisfy the first

\footnotetext{
${ }^{2}$ We note that Wiebel et al. [1] in the same context erroneously refer to Eq. (6) as the conservation of momentum law.
}

condition. Let $\mathbf{v}_{R}^{\lambda}$ and $\mathbf{v}^{\lambda}$ be two fields interpolated from the respective field above with $i \leq \lambda \leq j$. The linear interpolation for $\mathbf{v}_{R}^{\lambda}$ satisfies the following equation:

$$
\mathbf{v}_{R}^{\lambda}=\mathbf{v}_{R}^{i}+\frac{\lambda-i}{j-i}\left(\mathbf{v}_{R}^{j}-\mathbf{v}_{R}^{i}\right) \text {. }
$$

Then with the linearity of the divergence

$$
\operatorname{div} \mathbf{v}_{R}^{\lambda}=\operatorname{div} \mathbf{v}_{R}^{i}+\frac{\lambda-i}{j-i}\left(\operatorname{div} \mathbf{v}_{R}^{j}-\operatorname{div} \mathbf{v}_{R}^{i}\right)
$$

and with $\operatorname{div} \mathbf{v}_{R}^{i}=\operatorname{div} \mathbf{v}^{i}$ and $\operatorname{div} \mathbf{v}_{R}^{j}=\operatorname{div} \mathbf{v}^{j}$ it follows

$\operatorname{div} \mathbf{v}_{R}^{\lambda}=\operatorname{div} \mathbf{v}^{i}+\frac{\lambda-i}{j-i}\left(\operatorname{div} \mathbf{v}^{j}-\operatorname{div} \mathbf{v}^{i}\right)=\operatorname{div} \mathbf{v}^{\lambda}$.

The same considerations hold for the vorticity and yield curl $\mathbf{v}_{R}^{\lambda}=$ curl $\mathbf{v}^{\lambda}$. Thus, as mentioned above, the first condition holds for the interpolated fields too.

The second condition requires the potential flow's component normal to the boundary to be zero. Again, this condition does not depend on time and ensuring that it is satisfied for the time steps provided, is sufficient to guarantee that it holds for all instantaneous time steps. Note that the above considerations also imply that (1) and (2) hold for fields interpolated between potential fields of given time steps.

Summarizing this section, we find that the localized flow in the time-dependent case is obtained by computing the localized flow for the given time steps (as in the steady case) and linear interpolation between these time steps.

\section{A. Unsteady Compressible Flows}

For the steady case, we gave a modified Neumann problem to handle compressible flows in section IIIB. Unfortunately we are not able to give a modified Neumann problem for all unsteady flows. This is due to the fact that here $\frac{\partial \rho}{\partial t}$ does not necessarily vanish and thus the continuity equation (6) does not necessarily coincide with the compatibility condition (5).

However, compressibility does not always mean that condition (5) is not fulfilled. It is still possible to check whether the condition is fulfilled directly by evaluating the boundary integral. This check can be performed for the original Neumann problem or for the modified Neumann problem. If the condition is fulfilled for one of the systems one just solves this system to get the potential for the divergence and vorticity-free flow.

Additionally, in our experiments, we found that our implementation (which is described later in this paper) is very tolerant against small deviations from condition (5). The solutions are influenced only marginally by 
small deviations. However, we are working on a modified scheme for compressible unsteady flow with strongly time-varying density.

\section{INTERPRETATION OF THE FIELDS OF THE LOCALIZATION PROCESS}

So far we have only considered the mathematical construction of the region-specific flow. Along the way, some conditions were imposed to guarantee solvability of the problem. We will now dedicate some thoughts as to how these conditions affect applicability of our method to the general localized analysis of flows. We will also discuss the interpretation and use of the fields introduced in the previous sections. More datasetspecific results and application examples are given in Section VII.

\section{A. Scalar Potential}

The first field appearing during the localization process is the scalar potential obtained as solution of the Neumann problem. For the Hodge decomposition Polthier et al. [4] have used features in the scalar potential to identify features of the flow field. We will not go into further detail about the scalar potential as all interesting features also appear in the potential flow which, as mentioned, is the gradient of the scalar potential. Note additionally that all extrema of the scalar field lie on its boundary.

\section{B. Potential Flow}

From a feature oriented point of view the potential flow seems to be uninteresting. It has vanishing divergence and rotation and thus is irrotational and free of sinks and sources (saddles are possible, see the close-up in Fig. 2b). It is very simple, in fact it is the simplest flow (minimum of total kinetic energy, see [2]) matching the original inflow and outflow on the boundary of the considered region. Most of its behavior is determined by the geometry of the region, the rest is determined by the Neumann boundary conditions.

These properties, however, make it seem uninteresting only at first glance. The influence of the geometry of the region is very important. Engineers knowing the geometry of a region are able to predict the potential flow in the region with little effort. The potential flow shows how the flow would pass a region or object if viscosity and wall friction were negligible [2]. Thus it can be regarded as the most natural main flow direction.

As alluded to in the related work section, this property can be used to detect regions of reverse flow. Treating the direction suggested by the potential flow as main flow

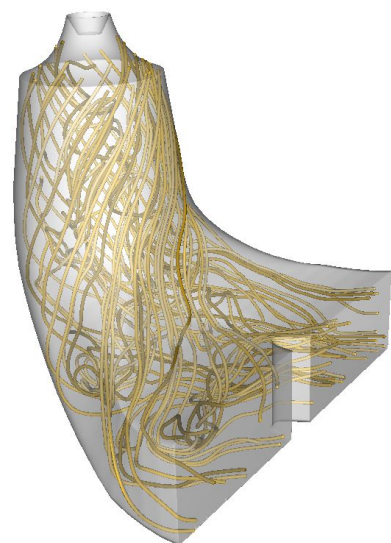

a) original flow

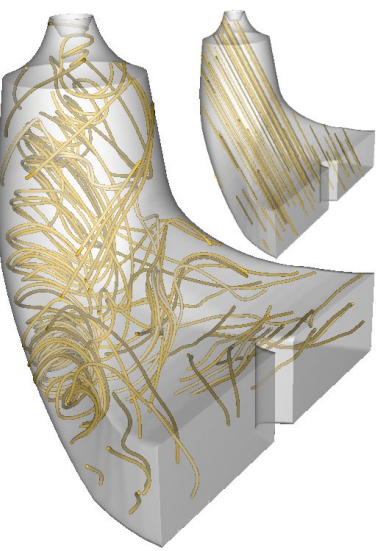

c) average flow and original flow with removed average flow

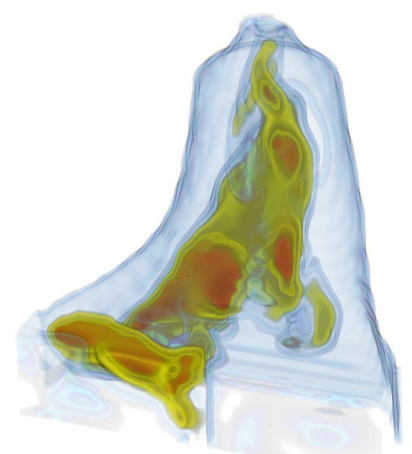

e) reverse flow region and strength

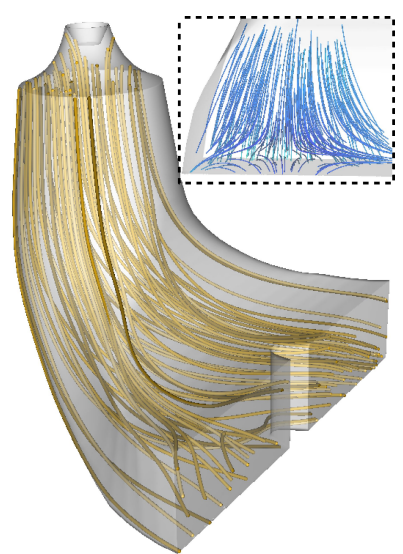

b) potential flow

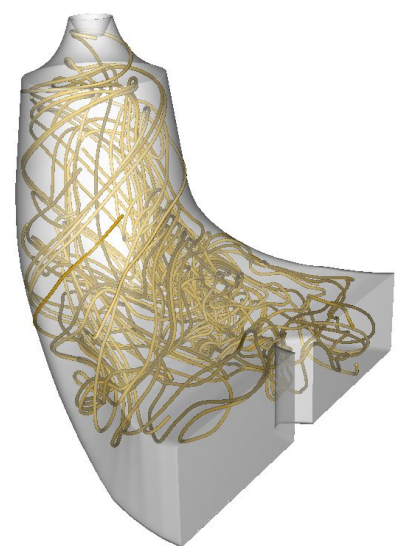

d) localized flow

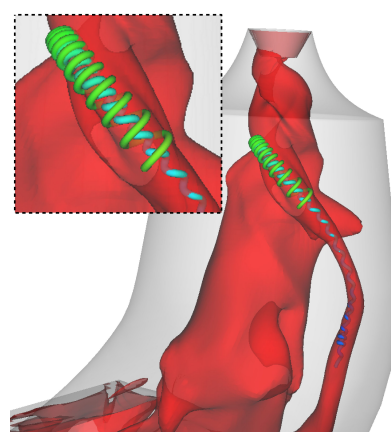

f) streamline in reverse flow region
Fig. 2. Image a) shows streamlines of the flow in a draft tube of a water turbine. Image b) shows how the potential flow follows the turn of the tube and a close up of the lower left corner. Images c) and d) demonstrate the advantages of subtracting potential flow field instead of constant average field. As the constant flow does not follow the tube, the flow with subtracted average often leads directly into the walls of the tube. The localized flow respects the boundary and does not lead into walls. In image e) a volume rendering shows locations and strength of reverse flow regions in the tube. Image f) shows an isosurface that represents the border of regions with reverse flow of another time step. A streamline in the original flow shows the reverse flow. 
direction we compute the dot product of the normalized original flow with the normalized potential flow. This yields a scalar value at every position which indicates whether the original flow has a component in (positive value) or against (negative value) the main flow direction. Then regions of reverse flow are easily identified as regions of negative value. Their borders can be visualized by isosurfaces of zero isovalue (see Figs. $2 \mathrm{f}$ and 4 ).

\section{Region-specific Flow}

From a purely physical point of view it does not seem feasible at first glance to manipulate a flow field in order to further its analysis. It is known practice, however, to decompose flow fields (see e.g. [2]) or to subtract a constant vector field to reveal structures that are not visible in the original field (heuristically, the average (boundary) flow is subtracted). The latter is justified by the principle of Galilean invariance which states that the properties of flow have to be the same for a constantly moving and for a resting observer. However, in most cases, this approach is not appropriate as it does not preserve boundary conditions. For example, in the flow around a stationary object (see Fig. 3) or through a channel (see Fig. 2), subtraction of a constant vector field yields streamlines that lead into the boundary surface. Since the boundary conditions are an integral part of the region-specific flow (via condition (2)), it does not suffer these problems.

Furthermore, both vorticity and divergence of the original flow are preserved in the region-specific approach. Therefore, feature definitions that build on these quantities and consequently algorithms that extract these features are naturally unaffected. Recently, Sadlo et al. [18] presented analysis and visualization of three-dimensional vector fields based on vorticity and vorticity lines. While the streamlines of the velocity are naturally different in the region-specific flow, the invariance of vorticity lines and hence the non-changing vorticity transport in the flow imply that all vortical structures are kept. This confirms our approach to be meaningful and to contain the information for the important features present in the original field. In summary, the region-specific flow contains exactly the local domain-specific contribution to the global flow.

As the localized flow is the difference of the original and the potential flow it represents the deviation of a particle in the original flow from the flow induced by the geometry. Consequently its magnitude is large where the influence of wall friction and viscosity are large and it is small where the original flow is nearly equal to the potential flow, i.e. laminar. We already mentioned that one can infer the potential flow mainly from the geometry of the region. Thus deviations from this flow are what is interesting in a flow. For the detection of these deviations the magnitude of the localized flow or better the ratio of the magnitude of the localized and the original flow are considered. For two-dimensional flows a simple color mapping of this ratio can give a first overview. Isosurfaces and direct volume rendering can be used for the same purpose when treating threedimensional flows (Figs. VII-A and 2).

Concerning the influence of the frame of reference on flow analysis, the region-specific flow delivers a natural abstraction. For the common case that features in the flow are obscured by a dominating constant flow, the influence of the latter is "caught" in the boundary conditions of the potential flow, even if it is non-constant. It is subsequently subtracted from the original flow and does not show up in the region-specific flow. By this, for the case of topological methods, critical points such as sinks, sources and spirals relating to extremal divergence and vorticity are much more likely to occur than in the original flow, enabling the use of such methods in a broader context of flow analysis.

Figure 3 exemplifies some of the previous considerations. The $2 \mathrm{D}$ vector field shown represents the incompressible flow passing around a cylinder. On the downstream side of the cylinder, the well known Kármán vortex street should develop. However, it cannot be observed in the original flow. Removing the numerically obtained average flow reveals some but not all of the features present and yields a strong diagonal flow component that has no physical interpretation. The constructed potential flow is very uniform except in the vicinity of the cylinder where it reflects the flow around it. Subtracting the potential flow from the original flow reveals all the downstream vortical structures by a topological analysis. In this example, subtracting the correct downstream component (a multiple of $\mathbf{e}_{x}=$ $(1,0)^{T}$ ) would also reveal all vortical structures and avoid the uninterpretable diagonal component. However, in practical applications (see Sec. VII-B) the original downstream component is often not known. In these cases the numerically obtained average vector was the best approximation up to now.

\section{Choice of Localization Region}

From the mathematics of solving the special Neumann problem 3 , it is only required that the subdomain $\Omega$ is open, bounded and connected. These requirements are easily fulfilled and do not constrain the choice of region much. Regarding the numerical schemes we apply 


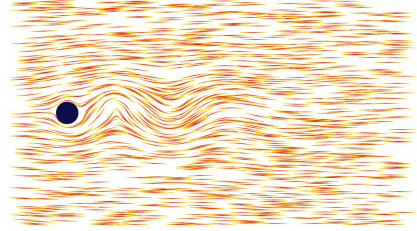

a) original flow

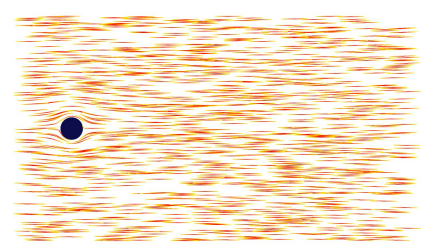

c) potential flow

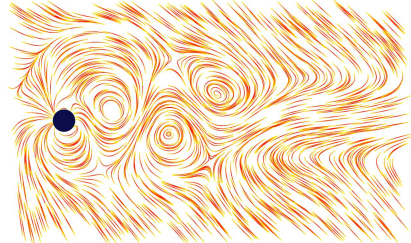

b) original flow minus average flow

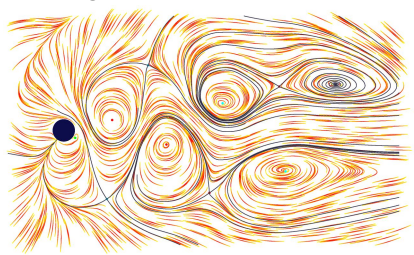

d) original flow minus potential flow

Fig. 3. Comparison of different fields obtained from cylinder data set with Kármán vortex street. a) Streamlines in the original flow. Only sinusoidal line structures adumbrate the vortices. b) Three vortices revealed by removing average flow. c) Potential flow induced by the flow on the boundary of the considered region. Note how the flow attaches to the cylinder and does not seem to cross it as it would be the case for constant average flow. d) Subtracting the potential flow reveals all five vortices present in the considered region by use of topology.

in the application of our ideas in the next section, a convex domain with piecewise smooth boundary is greatly beneficial in terms of convergence.

Choosing a localization region appropriately as input to the algorithm is the responsibility of the user. Often, an a-priori region of special interest can be a good choice and the engineers often know what is or should be interesting in their data sets. For the applications described in the previous subsection (reverse flow region detection and extraction of regions with large influence of friction and viscosity) the whole dataset is chosen for computation of the region-specific flow.

\section{E. Influence of Localization on $\lambda_{2}$-criterion}

To support our statement that the localized flow retains the essential features of the original flow, we discuss the influence of the localization on the $\lambda_{2}$-criterion in this section. The $\lambda_{2}$-criterion [13], as mentioned before, is a method for detecting vortex core regions. By this criterion a vortex core is defined as set of positions with low "modified" pressure, which means a set of positions where a certain matrix $S^{2}+\Omega^{2}$ derived from the local velocity gradient $\mathcal{J}=\nabla \mathbf{v}$ has two negative eigenvalues. The matrices $S$ and $\Omega$ are the symmetric and antisymmetric part of $\mathcal{J}$ :

$$
\mathcal{J}=S+\Omega=\frac{1}{2}\left(\mathcal{J}+\mathcal{J}^{T}\right)+\frac{1}{2}\left(\mathcal{J}-\mathcal{J}^{T}\right) .
$$

We now recall that the vorticity can be defined with the non-diagonal elements of the anti-symmetric part of the Jacobian $\mathcal{J}$ as follows:

$$
\operatorname{curl} \mathbf{v}=\nabla \times \mathbf{v}=\left(\Omega_{2,1}, \Omega_{0,2}, \Omega_{1,0}\right)^{T} .
$$

Since $\Omega$ is antisymmetric, all its diagonal elements are zero. Due to (9) and the antisymmetry all other elements are determined by the curl of $\mathbf{v}$. As the curl of the original and the region-specific flow are equal now, $\Omega$ is also equal for both flows. Unfortunately $S$ is only the same up to the sum of the diagonal elements as can be seen by

$$
\operatorname{tr}(\mathcal{J})=\operatorname{tr}(S)=\operatorname{div} \mathbf{v}
$$

and the fact that the divergence of the original and the region-specific flow are equal. However, this reasoning at least yields a strong similarity between $\lambda_{2}$ for the original and the localized flow. We, in fact, observed this similarity in all our experiments, see for example the left image of Fig. 4 for the EDELTA data set.

\section{IMPLEMENTATION}

In the following, we will revisit the construction of the region-specific flow from Section III and show how it can be achieved for discrete data sets.

We assume that the discrete flow field is given on the vertices of a simplicial (triangular or tetrahedral) grid, a form taken by many modern CFD data sets. We note that the derivation of the potential flow is basically independent of spatial dimension. In an implementation, however, differences show up since the method works on triangles in the two-dimensional case and on tetrahedra in three dimensions. By formulation in the context of finite element methods, a unified numerical formalism can be achieved nevertheless.

The region $\Omega$ is easily discretized as a connected subset of the original grid simplices. The Neumann problem is then discretized on this set by the application of a Galerkin-type finite element method. The basic idea is simple: by discretizing $u$ in a nodal basis $\left\{\phi_{i}\right\}$ that has one basis function for every grid point, Eqns. (3) and (4) can be written as a linear system

$$
A u=f \quad A \in \mathbb{R}^{n \times n} \text { and } u, f \in \mathbb{R}^{n},
$$

where $A$ is symmetric and sparse (cf. [19] for a very concise presentation of the general procedure).

The solution of this system is slightly more complicated than in the usual finite element case, owing to the fact that $A$ is not positive definite but positive semidefinite. This corresponds to the fact that the original problem (3) and (4) is only determined up to a 
constant. The compatibility condition (5) translates to

$$
\sum f_{i}=0
$$

implying that $f$ must be orthogonal to the kernel of $A$. If this discrete condition is fulfilled exactly, the commonly used Conjugate Gradients (CG) scheme can be employed to solve the singular system (10). Owing to inexactness in numerical integration, this is extremely difficult to ensure in practice, resulting in instability and extremely bad performance of the iterative scheme. Bochev and Lehoucq [20] give a possible solution to this class of problems: by reformulating the Neumann problem as a saddle point problem, a regularization approach can be employed to achieve stability and good convergence properties. Essentially, this results in a modified CG scheme that ensures that successive iterates remain outside the kernel of $A$. We found this approach both easily implemented and very stable.

Having obtained the discrete potential $u$, taking its gradient gives a cell-wise constant vector field. We use weighted averaging of neighboring simplices to compute the vector field values of $\mathbf{v}_{P}$ on the vertices of the grid. Finally, $\mathbf{v}_{R}$ is obtained by subtracting $\mathbf{v}_{P}$ from $\mathbf{v}$ at the grid vertices.

$A$ is best represented in a sparse storage format. This allows us to treat grids with millions of cells without resorting to out-of-core or cluster techniques which makes the implementation straightforward. Computational complexity is two-fold: the assembly of the system matrix $A$ is relatively costly since the complexity is linear in the number of grid cells. The complexity of the successive matrix inversion is then a function of the number of grid points and the smallest cell in-circle radius. The number of iterations of the CG scheme can be significantly reduced by application of preconditioning. Section VII$\mathrm{D}$ and Table I provide details on the performance of our implementation for a number of data sets.

Remark: As the matrix depends only on the grid and its connectivity, the same matrix can be used for all time steps in the unsteady case. Computing the matrix once and storing it for reuse avoids repeated costly computations and improves the performance of the localization procedure dramatically.

\section{RESUlTS AND EXAMPLES}

In this section we demonstrate the proposed techniques and show how they can help in furthering visualization on several application datasets. All data sets except the HART II dataset (which contains measured data) result from CFD simulations conducted in actual application research. In our analysis, we put a slight emphasis on topological methods and feature extraction schemes, since we believe that this class of methods is most benefitted by the localized flow approach.

The presented results were computed on a standard PC workstation with 3GB of RAM. Performance figures and dataset sizes are given in Table I.

\section{A. Delta Wing Configurations}

In the following we examine two datasets, both resulting from simulations of airflow around a single deltatype wing configuration (called EDELTA and TDELTA). Both datasets were computed in the context of numerical research into vortex breakdown by Markus Rütten at the DLR in Göttingen. Given on large unstructured adaptiveresolution grids, they present a serious challenge for visualization techniques in general owing to both performance issues and numerical stability problems. The datasets are quite large with $11 \mathrm{M}$ resp. $15 \mathrm{M}$ cells. Although they contain multiple time steps, we limit our analysis to single time slices in this paper and although both datasets exhibit vortex breakdown in later time steps, we also consider time steps that do not show it.

1) EDELTA: Figure 1 gives an overview of the localization process for this dataset. The original flow (left image) is dominated by a large (in magnitude) component induced by the original boundary condition. Choosing a box around the wing geometry for the localization, the potential flow (middle image) captures this component and essentially corresponds to a laminar flow around the wing. The region-specific flow looks interesting (right image). The primary vortical structures are clearly visible (the flow component along the vortex axes is essentially removed), as is the bow wave at the tip of the wing.

Next, we look at a time step that shows the vortex breakdown bubble above the wing. Theoretically, the breakdown bubble mainly consists of a recirculation zone that is shielded by two saddle points forcing the flow around it (for a more detailed exposition, see [22]). Figure 5 shows the results of a straightforward application of the Sujudi-Haimes algorithm to a spherical region around the breakdown bubble. The original vortex core is visible together with the breakdown saddle points (left image). The strongly curved region is an indication of the recirculation. However, the recirculation core is not cleanly extracted. The right image shows an identical visualization for the corresponding region-specific flow. While the critical points are unchanged, the recirculation is cleanly identified as a closed vortex core winding around the original vortex. We extract exactly the same structures as others working on this dataset [23] with 

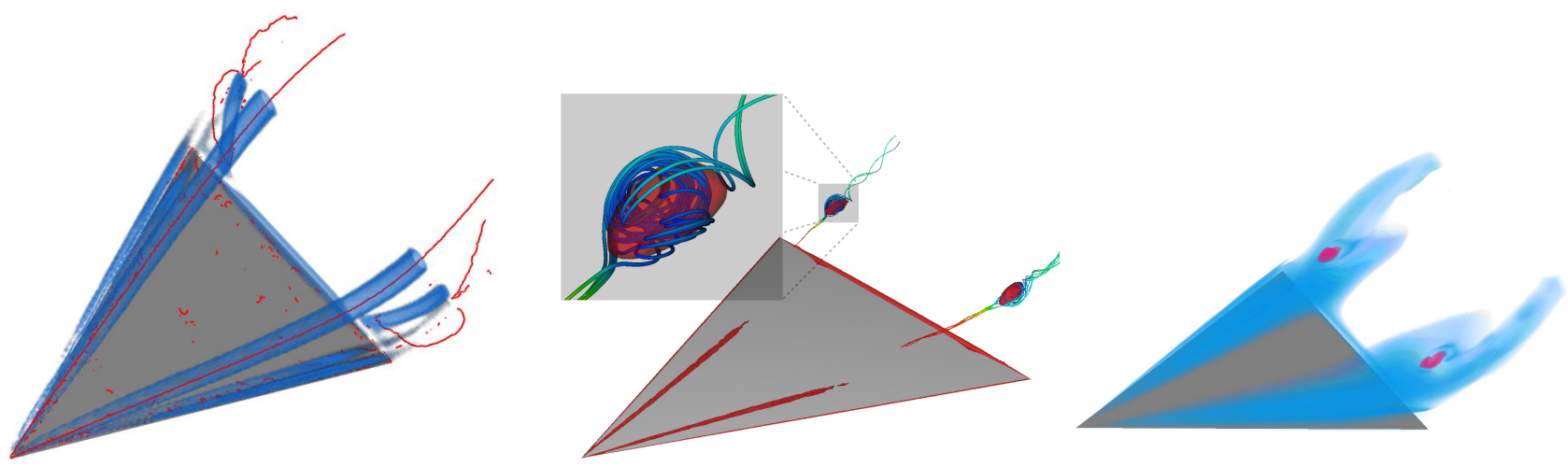

Fig. 4. Left: Vortex core lines and volume rendering of the $\lambda_{2}$-criterion, both computed for the localized flow around the delta wing of the EDELTA data set. The vortex core lines were extracted using the algorithm of Sujudi and Haimes in the parallel vectors version of Peikert and Roth [21]. Note how the volume rendering indicates even the secondary and tertiary vortices in the localized flow. Middle: Zero-isosurface (red) of dot product between original and potential flow indicating reverse flow regions. Streamlines in the image and the close-up show that the isosurfaces identify the recirculation in the vortex breakdown bubbles. Right: Volume rendering of the ratio of the velocity magnitude of original and localized flow. The opacity encodes the ratio while the color encodes the sign (positive blue, negative pink) of $(\mathbf{v} /\|\mathbf{v}\|) \cdot\left(\mathbf{v}_{P} /\left\|\mathbf{v}_{P}\right\|\right)$. In this representation we can see both, the recirculation zones and the extent of the vortices.

different methods. Essentially, the region-specific flow is much closer to an analytic breakdown bubble model, allowing for a clean identification of this phenomenon in this dataset.

To detect the recirculation in the same time step as above, but assuming not to know their location in advance, we used the potential flow as mentioned in Section V-B. The results can be seen in the middle and right image of Figure 4. The middle image shows a zeroisosurface of the dot product of the normalized original and normalized potential flow. It encloses the regions of reverse flow. The right image shows a volume rendering ${ }^{3}$ where the color represents the sign of the dot product. The opacity, however, represents the deviation of the original flow from the potential flow, i.e. the ratio of the magnitudes of original and localized flow. Both images show the recirculation zones quite clear. The right image additionally gives an impression of the deviation and thus of the influence of friction and viscosity. Both are large where the main vortices are located.

2) TDELTA: Although this simulation is quite similar to the EDELTA configuration, its spatial resolution is higher. This is especially true for the region close to the wing surface, making an analysis of the shear stress field feasible. One is especially interested in separation and attachment lines, whose extraction still poses major problems for modern datasets. In analogy to vortex core lines, separation and attachment lines can appear as part of the topological skeleton of the shear flow. Figure 6 (left) shows a LIC image of the original shear flow,

\footnotetext{
${ }^{3}$ Because of the complexity of the CFD grids we use the techniques introduced by Tricoche et al. [23].
}
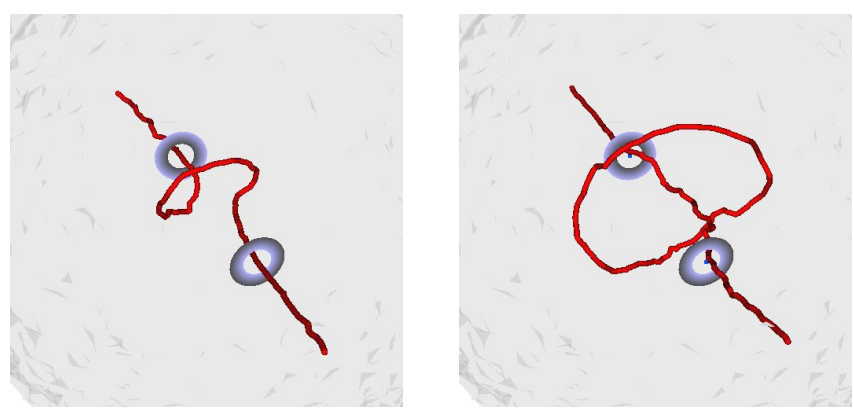

Fig. 5. Close-up of the vortex breakdown bubble. In the original flow, the main vortex core is strongly curved and distorted (left). In the region-specific flow, the recirculation type nature of the bubble is clearly identified by the closed vortex core (right image). Critical points are in this case unaffected by the localization.

overlaid with its topological graph. The high number of critical points is a result of the numerically unstable shear flow computation that involves numerical derivatives. Some separatrices are indicative of separation/attachment behavior, however, the picture is incomplete. Using the entire wing as localization region, the resulting regionspecific flow shows all the features as part of its topology (middle image). Since the subtracted potential flow is very smooth, the localized shear flow does still contain significant amounts of numerical noise. In spite of this, the extracted separation and attachment lines are of a very good quality and match the original shear flow properties (right image). In analogy to the vortex cores in the EDELTA dataset (cf. [1]), by the use of regionspecific flow, it is possible to extract important features easily as part of the topological graph. 

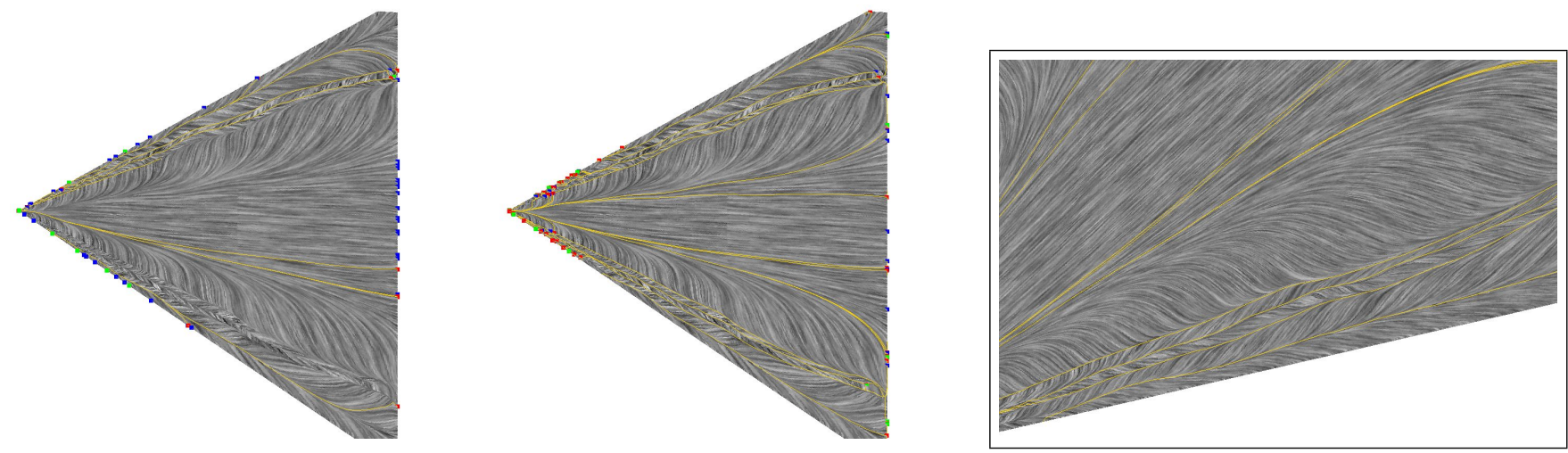

Fig. 6. Topology of the surface shear stress in the TDELTA dataset. The topology of the original shear flow captures some features, but is incomplete (left). All separation and attachment lines appear in the topology of the localized shear flow (middle). All images also show a LIC representation of the original shear flow. A close-up reveals a perfect match between original shear flow and localized features (right).

\section{B. HART II}

The HART II dataset does not result from a simulation. It consists of measurements of a helicopter rotor blade wake (cf. [24]), aiming at improved knowledge about the evolution of the vortices generated by the wake and reducing the rotor noise which is created by interaction of the wake and a following blade hitting the wake. PIV (Particle Image Velocimetry) was used to obtain instantaneous flow field data in a large observation area and in a smaller close-up view of the vortex core. By nature, PIV produces measurements on $2 \mathrm{D}$ slices of the three-dimensional flow field. We consider one such slice with $8 \mathrm{~K}$ vertices that cuts through the wake and thus the wake vortices of one rotor blade.

This type of dataset is of special interest since the correct frame of reference is unclear: while the observer is static, the rotor blades are moving. From the left image of Fig. 7, showing the original measured data, no vortical structure can be inferred. Without making any assumptions about the correct frame of reference and using the entire measurement domain as localization region, the structures of primary interest are easily extracted from the region-specific flow using simple topological tools. The two wake vortices present in the flow are clearly visible in the right image. The stronger vortex stems from the last passing blade while the origin of the smaller vortex is the tip of a blade passing earlier. The latter vortex is smaller due to two reasons: it is older and thus decaying and it was hit by the last passing blade (this disturbs its vortical nature). The goal of the engineers, which is to determine the position of the wake vortices, is easily achieved with the region-specific flow.
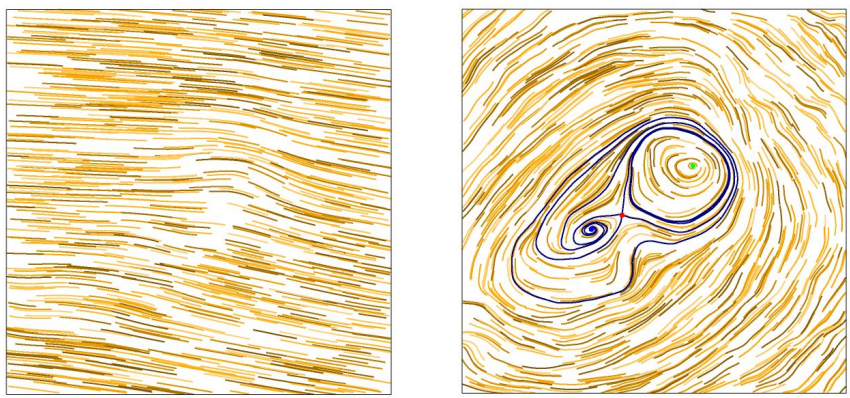

Fig. 7. HART II data set consisting of PIV measurements of helicopter rotor blade wake. Left image shows original measured flow in a plane cutting through the wake. The wake vortices of the passing blades are not visible. On the right, the topology of the region-specific flow reveals the vortices present in the correct frame of reference.

\section{Draft Tube}

This dataset represents the draft tube of a Francis turbine, in which the runner is spinning in the inlet part of the turbine (see Fig. 2a). The runner (at the top) induces a spinning motion in the water, which leaves the turbine (bottom) after passing through the curved tube. We essentially used this dataset to illustrate the unsuitability of the average (boundary) flow in the localization of such datasets.

From Figure 2 it is obvious that the average flow fails to approximate the overall boundary flow due to the strong curved nature of the domain (image c). The potential flow, however, follows the curved shape of the tube (image b). Subtracting the average flow and the potential flow from the original flow (images $\mathrm{c}$ and d), we observe that the localized flow does not violate the boundary condition on the tube wall, as opposed to the average-reduced flow. In summary, the region-specific flow shows a more natural behavior. 
For this dataset the detection of reverse flow as proposed by Laramee et al. [17] must fail because the main flow direction turns by 90 degrees while passing through the tube. In contrast, our method using the dot product of original and potential flow detects the reverse flow easily (Fig. 2f). The volume rendering in Fig. 2e even allows to get an impression of how strong the flow direction differs from the main flow direction. The difference, i.e. the negative dot product of the normalized vectors of original and potential flow, increases from yellow to red. The bluish area shows the space surrounding the reverse flow for completeness.
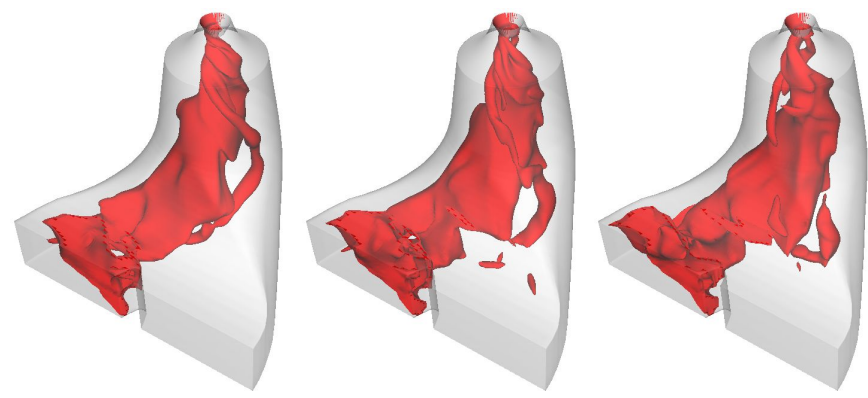

Fig. 8. Three snapshots of an animation showing the movement of reverse flow in tube dataset. Notice the movement and split of the reverse flow region in the main vortex as long thin parts of the isosurface.

The extension of the localized flow approach to unsteady flows allows us to visualize the evolution of the reverse flow regions as shown in Figure 8.

\section{Performance}

An overview of the performance of the localization for steady flows (or single time steps) is given in Table I, both for datasets we discussed here as well as for other datasets available to us. The timings given there reflect the entire procedure of extracting a subregion, solving for the potential, computing its gradient and subtracting the obtained potential flow. For small to medium sized datasets the timings indicate an easy incorporation of the localization procedure in a typical visualization workflow. For large datasets this is not possible because of the large computation time which is due to the complexity of the numerical schemes involved. However, the large computation time is only a minor fraction of the original simulation effort, and can therefore go almost unnoticed.

\section{CONCLUSION AND OUTLOOK}

We have presented a method to isolate the flow in subdomains of flow data sets from the flow in the neighborhood by constructing an irrotational and divergencefree field from the flow at the boundary of the subdomain and subtracting it from the original field. We have extended this method to unsteady flow fields. Furthermore, we have given applications of components (localized and potential) of the original flow. While the potential flow can be used to detect reverse flow regions, the magnitude of the localized flow can reveal regions where the influence of friction dominates the flow.

The localization retains the original features of the flow and is thus ready for the application of many standard methods for feature and topology analysis. We detailed this by examining the influence of the localization on topology, vortex core line extraction and the $\lambda_{2}$-criterion. We discussed the differential equation that has to be solved to obtain the potential flow fields and described our implementation. Applied to large data sets from CFD simulations and to a measurement data set our method proved to be scalable and robust.

Future work may include the following research avenues:

- Having the time-dependent extension of the localized flow it may be interesting to track reverse flow regions over time to learn more about their creation and evolution. Additionally, the interpretation of path lines in the localized flow is an open question which we will address in the the future.

- As solving for the potential $u$ includes handling of very large matrices we intend to implement a parallel solver to reduce the computation time.

\section{ACKNOWLEDGMENTS}

The authors would like to thank Markus Rütten, Hugues Richard and Berend van der Wall from German Aerospace Center (DLR) in Göttingen and Braunschweig, as well as VA Tech Hydro and Ronald Peikert from ETH Zürich for providing the data sets. The discussions with Markus Rütten also were of great help for the time-dependent case. We would especially like to thank Xavier Tricoche for many constructive remarks and interesting discussions on the topic. Thanks also go to all FAnToM development group members for their implementation efforts. This work was partly supported by DFG grants HA 1491/15-4, HA 1491/15-5 and SCHE 663/3-7.

\section{APPENDIX I}

As mentioned before, one reviewer raised concerns about the notion of rotation and divergence being local in nature. We give his example problem here, as we think that discussing it may yield deeper insight in the idea of localized flow. 


\begin{tabular}{|l|r|r|r|r|}
\hline Region & Dimension & \# Simplicial Cells & \# Vertices & Computation Time in sec. \\
\hline \hline Box around cylinder & 2D & $4.7 \mathrm{~K}$ & $4.8 \mathrm{~K}$ & 0.66 \\
HART II & 2D & $16.6 \mathrm{~K}$ & $8.5 \mathrm{~K}$ & 0.49 \\
TDELTA (wing) & 2D & $156 \mathrm{~K}$ & $81 \mathrm{~K}$ & 10.2 \\
\hline Ball & 3D & $202.6 \mathrm{~K}$ & $36.5 \mathrm{~K}$ & 7.37 \\
Box in furnace chamber & 3D & $540.0 \mathrm{~K}$ & $964.4 \mathrm{~K}$ & 22.03 \\
Ball with hole & 3D & $1.1 \mathrm{M}$ & $0.4 \mathrm{M}$ & 119.09 \\
Draft Tube & 3D & $4.9 \mathrm{M}$ & $0.9 \mathrm{M}$ & 322.07 \\
ICE train & 3D & $6.2 \mathrm{M}$ & $1.1 \mathrm{M}$ & 381.16 \\
EDELTA (box) & 3D & $17.3 \mathrm{M}$ & $3.0 \mathrm{M}$ & 1386.07 \\
TDELTA (entire) & 3D & $25.8 \mathrm{M}$ & $4.5 \mathrm{M}$ & 3403.10 \\
\hline
\end{tabular}

TABLE I

PERFORMANCE FIGURES FOR LOCALIZED FLOW PROCEDURE OF DIFFERENT DATA SETS

The reviewer stated that a slow moving large vortex would provide a counter-example to the locality argument (especially for rotation).

For the discussion of his problem, at first it should be noted that the mathematical definition of divergence and rotation (curl) is local in nature and that this is what we refer to in the whole paper. We know that there exists vorticity transport in flows and thus the rotation can change by global influences. However, as we will detail in the following, we do not believe that this influences the soundness of our method for the moving vortex and other problems.

Rotation and divergence of the region-specific flow $\mathbf{v}_{R}$ are identical to the original flow $\mathbf{v}$ inside the domain $\Omega$ in the sense of the mathematical definition. This is condition (1) in section III and the essence of the whole construction. It follows by Stokes' theorem that e.g. the circulation around the boundary curve of any surface inside $\Omega$ also coincides:

$$
\begin{aligned}
C_{R} & =\int_{\partial S} \mathbf{v}_{R} d x \\
& =\int_{S} \operatorname{curl} \mathbf{v}_{R} \cdot \mathbf{n} d S \\
& =\int_{S} \operatorname{curl} \mathbf{v} \cdot \mathbf{n} d S \\
& =\int_{\partial S} \mathbf{v} d x \\
& =C .
\end{aligned}
$$

In this mathematical sense, the localized flow captures the rotation and divergence of the global flow inside the chosen region. Of course, the localized flow does not capture additional rotation or divergence outside the chosen region. In the moving-vortex-example, the localized flow will only be influenced by the rotation of the large vortex inside the chosen region. Any rotation outside that region will not show up in the localized flow, so that the region determines whether the localized flow gives a complete or a partial picture of the large vortex. If, for example, the region is fixed and the area essentially influenced by the vortex moves over time from inside the region to the outside, the localized flow will show a smaller and smaller part of the vortex. In our eyes, this limitation is obvious to any fluid dynamics researcher or engineer and will no disturb him. We believe so because the construction of the localized flow by separating a divergence-free potential flow from the remaining part uses very well known basic concepts of fluid dynamics. If one wants to examine the whole moving vortex using localized flow one has to choose a region that covers the whole path of the vortex. As the examples in our paper show, it even makes sense to choose the whole simulation domain for computing the localized flow in many cases. The draft tube is an example of how the localized flow can be used to determine reverse flow regions in a flow with a moving vortex.

\section{REFERENCES}

[1] A. Wiebel, C. Garth, and G. Scheuermann, "Localized Flow Analysis of 2D and 3D Vector Fields," in Data Visualization 2005: Proceedings of Eurographics/IEEE-VGTC Symposium on Visualization 2005 (EuroVis 2005), K. Brodlie, D. Duke, and K. Joy, Eds. Eurographics Association, June 2005, pp. 143 150.

[2] G. K. Batchelor, An Introduction to Fluid Dynamics. Cambridge University Press, 1967, ch. 2.7, 6.1 and 6.2.

[3] K. Polthier and E. Preuß, "Variational Approach to Vector Field Decomposition," in Proc. of Eurographics Workshop on Scientific Visualization, R. van Liere, F. Post, and et. al., Eds. Springer Verlag, 2000. 
[4] _ , "Identifying Vector Field Singularities Using a Discrete Hodge Decomposition," in Visualization and Mathematics III, H. C. Hege and K. Polthier, Eds. Springer Verlag, 2003, pp. $113-134$.

[5] Y. Tong, S. Lombeyda, A. Hirani, and M. Desbrun, "Discrete Multiscale Vector Field Decomposition," ACM Transactions on Graphics (TOG), vol. 22, no. 3, pp. 445 - 452, July 2003.

[6] F. H. Post, B. Vrolijk, H. Hauser, R. S. Laramee, and H. Doleisch, "The State of the Art in Flow Visualization: Feature Extraction and Tracking," Computer Graphics Forum (Blackwell CGF), vol. 22, no. 4, pp. 775 - 792, 2003.

[7] J. L. Helman and L. Hesselink, "Representation and Display of Vector Field Topology in Fluid Flow Data Sets," IEEE Computer, vol. 22, no. 8, pp. 27 - 36, August 1989.

[8] A. Globus, C. Levit, and T. Lasinski, "A Tool for Visualizing the Topology of Three-Dimensional Vector Fields," in Proceedings of the 2nd conference on Visualization '91, October 1991, pp. $33-40$.

[9] G. Scheuermann, H. Krüger, M. Menzel, and A. P. Rockwood, "Visualizing Nonlinear Vector Field Topology." IEEE Trans. Vis. Comput. Graph., vol. 4, no. 2, pp. 109 - 116, 1998.

[10] X. Tricoche, T. Wischgoll, G. Scheuermann, and H. Hagen, "Topology Tracking for the Visualization of Time-Dependent Two-Dimensional Flows," Computers \& Graphics, vol. 26, no. 2, pp. $249-257,2002$.

[11] H. Theisel, T. Weinkauf, H.-C. Hege, and H.-P. Seidel, "Saddle Connectors - An Approach to Visualizing the Topological Skeleton of Complex 3D Vector Fields," in Proceedings of the conference on Visualization '03, H. Rushmeier, G. Turk, and J. J. van Wijk, Eds., 2003, pp. 225 - 232.

[12] D. Sujudi and R. Haimes, "Identification of Swirling Flow in 3-D Vector Fields," in 12th AIAA CFD Conference, San Diego CA, June 1995

[13] J. Jeong and F. Hussain, "On the Identification of a Vortex," Journal of Fluid Mechanics, vol. 285, pp. 69 - 94, 1995.

[14] J. Sahner, T. Weinkauf, and H.-C. Hege, "Galilean Invariant Extraction and Iconic Representation of Vortex Core Lines," in Data Visualization 2005: Proceedings of Eurographics/IEEEVGTC Symposium on Visualization 2005 (EuroVis 2005), K. Brodlie, D. Duke, and K. Joy, Eds. Eurographics Association, June 2005, pp. $151-160$.

[15] S. Stegmaier, U. Rist, and T. Ertl, "Opening the Can of Worms: An Exploration Tool for Vortical Flows," in Proceedings of IEEE Visualization '05, C. Silva and E. Gröller and H. Rushmeier, Ed. IEEE, 2005, pp. 463-470.

[16] D. C. Banks and B. A. Singer, "Vortex Tubes in Turbulent Flows: Identification, Representation, Reconstruction," in IEEE Visualization '94, R. D. Bergeron and A. E. Kaufman, Eds. IEEE Computer Society, 1994, pp. 132-139.

[17] R. S. Laramee, C. Garth, H. Doleisch, J. Schneider, H. Hauser, and H. Hagen, "Visual Analysis and Exploration of Fluid Flow in a Cooling Jacket." in IEEE Visualization. IEEE Computer Society, 2005, pp. $623-630$.

[18] F. Sadlo, R. Peikert, and E. Parkinson, "Vorticity Based Flow Analysis and Visualization for Pelton Turbine Design Optimization," in Proceedings of the IEEE Visualization '04 (VIS '04), G. Turk, J. J. van Wijk, and R. Moorhead, Eds. IEEE, October 2004, pp. $179-186$.

[19] J. Alberty, C. Carstensen, and S. A. Funken, "Remarks Around 50 Lines of Matlab: Short Finite Element Implementation," Numerical Algorithms, vol. 20, pp. 117 - 137, 1999.

[20] P. Bochev and R. B. Lehoucq, "On the Finite Element Solution of the Pure Neumann Problem," SIAM Review, vol. 47, no. 1, pp. $50-66,2005$.

[21] R. Peikert and M. Roth, "The "Parallel Vectors" Operator A Vector Field Visualization Primitive," in Proceedings of the conference on Visualization '99: celebrating ten years, 1999, pp. $263-270$.

[22] C. Garth, X. Tricoche, T. Salzbrunn, T. Bobach, and G. Scheuermann, "Surface Techniques for Vortex Visualization," in Data Visualization 2004 - Eurographics/IEEE TCVG Symposium on Visualization Proceedings, O. Deussen, C. Hansen, D. A. Keim, and D. Saupe, Eds. Konstanz, Germany: Eurographics Association, May 2004, pp. 155 - 164.

[23] X. Tricoche, C. Garth, G. Kindlmann, E. Deines, G. Scheuermann, M. Ruetten, and C. Hansen, "Visualization of Intricate Flow Structures for Vortex Breakdown Analysis," in Proceedings of the IEEE Visualization 2004 (VIS'04), H. Rushmeier, G. Turk, and J. J. van Wijk, Eds. IEEE Computer Society, October 2004, pp. 187 - 194.

[24] B. G. van der Wall, C. L. Burley, Y. Yu, H. Richard, K. Pengel, and P. Beaumier, "The HART II Test - Measurement of Helicopter Rotor Wakes," Aerospace Science and Technology, no. 8, pp. $273-284,2004$.

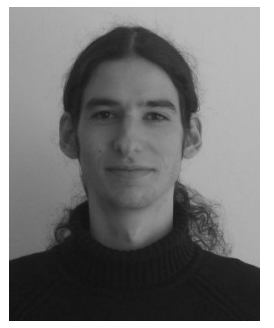

Alexander Wiebel received the Dipl.-Inf. (MS in computer science) degree in 2004 from the University of Kaiserslautern. Currently, he is working as a research assistant and $\mathrm{PhD}$ student at the Department of Computer Science of the University of Leipzig. His research interests include flow visualization, visualization systems, feature-base visualization and visualization of time-dependent vector fields.

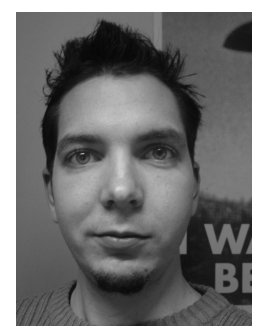

Christoph Garth received the Diplom (MS) degree in mathematics and computer science in 2003 from the University of Kaiserslautern. Currently, he is working as a $\mathrm{PhD}$ student in the Computer Graphics \& Visualization Group at the University of Kaiserslautern. His research interests include computer graphics, visualization systems and scientific visualization of vector fields and simulation data.

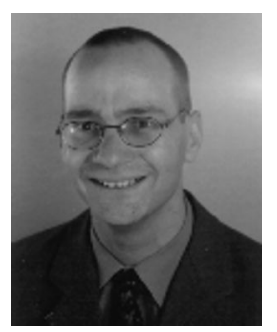

Gerik Scheuermann received the BS and MS degrees in mathematics in 1995 from the University of Kaiserslautern. In 1999, he received the PhD degree in computer science, also from the University of Kaiserslautern. During 19951997, he conducted research at Arizona State University for about a year. He worked as a postdoctoral researcher at the Center for Image Processing and Integrated Computing (CIPIC) at the University of California, Davis, in 1999 and 2000. Between 2001 and 2004, he was an assistant professor for computer science at the University of Kaiserslautern. Currently, he is a professor in the Computer Science Department of the University of Leipzig. His research topics include algebraic geometry, topology, Clifford algebra, image processing, graphics, and scientific visualization. $\mathrm{He}$ is a member of the ACM, IEEE Computer Society, and GI. 